\title{
THE DIAMETER OF THE GRAPH OF A SEMIRING
}

\author{
ANASTASE NAKASSIS
}

ABSTRACT. In this paper, it is proven that the diameter of the graph of a semiring with more than two elements does not exceed three.

Introduction. Y. F. Lin and J. S. Ratti [1], [2], [3] studied the graphs of semirings and conjectured that:

(a) The graph of a semiring that contains more than two elements is connected.

(b) The diameter of the above-mentioned graph does not exceed three. Starting from the results they obtained, we are going to prove that both of these conjectures are correct.

To clarify the terms of this paper, we will first review some basic definitions:

A semiring is a nonempty set equipped with two operations: addition, +, and multiplication (denoted by juxtaposition), such that addition is associative and commutative and multiplication associative and distributive across addition both from the left and the right.

Let $R$ be a semiring, $U(R)$ the set of all proper subsemirings of $R$, and $G(R)=(U(R), E)$ the nonoriented graph whose edges are the couples $(A, B)$ that fulfill the relationship $A \cap B \neq \varnothing$ (with the understanding that $A$ and $B$ are elements of $U(R)) . G(R)$ is said to be the graph of $R$.

The distance between two vertices $A, B$ of a graph, $d(A, B)$, is the number of edges in a shortest path between $A$ and $B$. (We define $d(A, B)$ to be 0 if $A=B$ and $+\infty$ if no path joins $A$ to $B$.) The diameter $D(G)$ of a graph $G$ is the supremum of $d(A, B)$ when $A$ and $B$ run over the vertices of the graph. We are going to examine the second conjecture. It is evident that if it is true, so is the first one.

The diameter of the graph of a semiring. The main results of [1] and [2] may be summarized in

THEOREM 1. The diameter of $G(R)$ does not exceed 3 if card $R>2$ and one of the following conditions is fulfilled:

$\left(\mathrm{a}_{1}\right) R$ contains a left unit.

$\left(\mathrm{a}_{2}\right) R$ contains a right unit.

(b) $R$ is commutative.

Received by the editors March 23, 1973 and, in revised form, February 17, 1976. AMS (MOS) subject classifications (1970). Primary 05C25, 16A78. 
Proof. See [1] and [2].

We shall try to prove that the hypothesis "the diameter of $G(R)$ exceeds 3 and card $R>2$ " is self-contradictory. We shall therefore assume in Lemmas 1-7 that $R$ is a semiring such that the diameter of $G(R)$ exceeds 3 and that card $R>2$. In view of Theorem 1 , this immediately implies that $R$ is not commutative and that it does not contain a left or right unit. Moreover:

LEMMA 1. (a) For any $a \in R$, the semiring $\langle a\rangle$ that is generated by $a$ is proper [otherwise, $R$ is commutative].

(b) No element of $R$ fulfills the double equality $x R=R x=R$ [otherwise $R$ contains a unit].

(c) For any $y$ and $z$ that belong to $R, y R$ and $R z$ are subsemirings of $R$ (not always proper) and their intersection is not void [both contain $y z$ ].

Lemma 2. Let $d(A, B)>3$. Then either

$$
\begin{aligned}
& x R=R \text { and } R x \neq R \text { for all } x \in A \cup B \text { or } \\
& x R \neq R \text { and } R x=R \text { for all } x \in A \cup B .
\end{aligned}
$$

Proof. For any $a \in A, b \in B$, the sequences $\{A, a R, R b, B\}$ and $\{A, R a, b R$, $B$ ) are not paths in $G(R)$ since $d(A, B)>3$. Using Lemma 1(b), we deduce that either

(1) $a R=b R=R$, or

(2) $R a=R b=R$.

Suppose that $a R=b R=R$ (case (2) is the dual of (1) and can be treated in a similar way), then $R a \neq R$ and $R b \neq R$. By fixing $a$ and letting $b$ run over $B$, or by fixing $b$ and letting $a$ run over $A$, we obtain that for any $x$ belonging to $A \cup B, x R=R$ and $R x \neq R$.

Since the diameter of $G(R)$ exceeds 3 , there is a couple $(A, B)$ such that $d(A, B)>3$. Fix $A$ and $B$ in what follows (Lemmas 3-7) and suppose that for all $x \in A \cup B, x R=R$ and $R x \neq R$ (by Lemma 2, this may be done without loss of generality). Then,

Lemma 3. For any $a \in A$ and $b \in B, R a+R b=R$.

Proof. Let $\left\langle a^{2}, b^{2}\right\rangle$ be the subsemiring of $R$ which is generated by $a^{2}$ and $b^{2}$. By hypothesis, $d(A, B)>3$. Therefore, $\left\{A,\left\langle a^{2}, b^{2}\right\rangle, B\right\}$ cannot be a path. Thus, $\left\langle a^{2}, b^{2}\right\rangle=R$. On the other hand, inspection of the elements of $\left\langle a^{2}, b^{2}\right\rangle$ shows that $\left\langle a^{2}, b^{2}\right\rangle$ is a subset of $R a \cup R b \cup(R a+R b)$. So $R a \cup R b$ $\cup(R a+R b)=R$.

If $a$ belonged to $R a, R$ would contain a left unit (by hypothesis, $a R=R$ ); if $a$ belonged to $R b, d(A, B)$ would be at most 2 , since $\{A, R b, B\}$ would be a path of length two (by hypothesis, $R b \neq R$ ). So $a$ belongs to $R a+R b$ and $R a \subseteq R a+R b$. Similarly, $R b \subseteq R a+R b$. So, $R a+R b=R$. Let us choose once and for all an $a \in A$ and a $b \in A$ which will remain fixed in Lemmas 4-7. 
LEMMA 4. Let

$$
C_{a}=\{z \mid(z+R a) \cap\langle a\rangle \neq \varnothing\}, \quad C_{b}=\{z \mid(z+R b) \cap\langle b\rangle \neq \varnothing\} .
$$

Then, $C_{a}=C_{b}=R$.

Proof. $C_{a}$ and $C_{b}$ are subsemirings of $R$. Indeed, if $z$ and $z^{\prime}$ are in $C_{a}$,

$$
z+r a=p(a) \text { and } z^{\prime}+r^{\prime} a=p^{\prime}(a)
$$

for some $r$ and $r^{\prime}$ in $R, p(a)$ and $p^{\prime}(a)$ in $\langle a\rangle$. Then

$$
\left(z+z^{\prime}\right)+\left(r+r^{\prime}\right) a=p(a)+p^{\prime}(a)
$$

and since $\langle a\rangle$ is commutative,

$$
\begin{aligned}
z z^{\prime}+\left(z r^{\prime}+r p^{\prime}(a)\right) a & =z\left(a^{\prime}+r^{\prime} a\right)+r p^{\prime}(a) a=z p^{\prime}(a)+r p^{\prime}(a) a \\
& =(z+r a) p^{\prime}(a)=p(a) p^{\prime}(a) .
\end{aligned}
$$

Consequently, $z+z^{\prime}$ and $z z^{\prime}$ belong to $C_{a}$, which is therefore a subsemiring of $R$. By the same argument, we prove $C_{b}$ to be a subsemiring. Since $R a+R b$ $=R b+R a=R, a=c a+d b, b=c^{\prime} a+d^{\prime} b$, for some $c, c^{\prime}, d$ and $d^{\prime}$ in $R$. Then $R b \cap C_{a}$ contains $d b$ and $R a \cap C_{b}$ contains $c^{\prime} a$.

The sequences $\left\{A, C_{a}, R b, B\right\}$ and $\left\{A, R a, C_{b}, B\right\}$ cannot be paths $(d(A, B)$ $>3)$. Therefore, $C_{a}=C_{b}=R$.

LEMMA 5. For any $x \in R,\langle x\rangle+R=R$.

Proof. $\langle x\rangle+R$ is a subsemiring of $R$. Since $x$ belongs both to $C_{a}$ and $C_{b}$, $\langle x\rangle+R$ meets $\langle a\rangle$ as well as $\langle b\rangle$. So if $\langle x\rangle+R \neq R,\{A,\langle x\rangle+R, B\}$ would be a path of length 2 , a contradiction.

LEMMA 6. $R$ contains an element $w$ such that

(1) $x=x+w$ for all $x \in R$,

(2) $x w=w x=w$ for all $x \in R$.

Proof. Since $a R=b R=R$ for some well-chosen $u$ and $v$ in $R, a u=a$ and $b v=b$. Then, $\langle a+u\rangle+R \subseteq a+R$ and $\langle b+v\rangle+R \subseteq b+R$. Indeed, any power of $(a+u)$ contains, if developed, a term of the form $a u u \cdots u=a$. Using Lemma 5, we deduce that $a+R=b+R=R$, and more generally that

$$
a^{i}+R=b^{j}+R=R \text { for any } i=1,2, \ldots \text { and } j=1,2, \ldots
$$

Since $a+R=R$, there is a $w$ such that $a+w=a$. It is easily seen that $x+w=x$ for all $x \in R$ and that $w$ is therefore unique. Take the relation $a^{2}+R=R$. Then

$$
a^{2}+a w=a(a+w)=a^{2} \text { and } a^{2}+w a=(a+w) a=a^{2} .
$$

By virtue of the equality $a^{2}+R=R$, for all $x \in R, x+a w=x+w a=x$, 
and since $w$ is unique, $w=w a=a w$. For the same reasons, $w b=b w=w$.

Since $\left\langle a^{2}, b^{2}\right\rangle=R$ (see Lemma 3), we deduce that $w x=x w=w$ for all $x \in R$.

LEMMA 7. $a=w$ or $b=w$.

Proof. $\{A,\langle a, w\rangle,\langle b, w\rangle, B\}$ is not a path and hence either $\langle a, w\rangle=R$ or $\langle b, w\rangle=R$. Let $\langle a, w\rangle=R$. This assumption entails no loss of generality, since the case $\langle b, w\rangle=R$ can be similarly treated. It is easily seen that $\langle a, w\rangle=\langle a\rangle \cup\{w\}$ and since $A \cap B=\varnothing, b$ does not belong to $\langle a\rangle$ and $b=w$. Since $b R=R, R=w R=\{w\}$.

Lemma 7 contradicts our original assumption that $\operatorname{card} R>2$. So:

THEOREM 2. When $R$ has more than two elements, the diameter of $G(R)$ does not exceed three.

Corollary. $G(R)$ is connected if card $R>2$.

REMARKS. (1) The proof of Theorem 2 may be substantially simplified if $R$ is uncountable or finite.

In the first case, the proof is straightforward. If $A$ and $B$ are two proper subsemirings of $R$, either $A \cap B \neq \varnothing$ and $d(A, B)$ is 0 or 1 , or $A \cap B=\varnothing$. In the latter case, let $a \in A$ and $b \in B$. Then $\langle a, b\rangle$ is countable or finite. Hence $\langle a, b\rangle \neq R$ and $\{A,\langle a, b\rangle, B\}$ is a path of length 2 .

If $R$ is finite, every subsemiring of $R$ contains one or more elements satisfying the double equality $x^{2}=x+x=x$.

Indeed, let $D$ be a subsemiring of $R$. Consider an element $z$ in $D$ and the sequence $\{n z\}$. Since $D$ is finite, there exist positive integers $k$ and $k^{\prime}$ such that $k z=\left(k+k^{\prime}\right) z$. Let $y=k k^{\prime} z$. Then we may prove that $y+y=y$. (Write the first $y$ as the sum of $k^{\prime}$ terms, each equal to $k z$, and the second as the sum of $k$ terms, each equal to $k^{\prime} z$. Then use the associative law to eliminate the last $k$ terms.)

We also remark that if $n$ is an integer greater than 1 ,

$$
y^{n}+y^{n}=y^{n-1}(y+y)=y^{n-1} y=y^{n} .
$$

Consider now the sequence $\left\{y^{n}\right\}$. Since $D$ is finite for some positive integers $i$ and $j, y^{i}=y^{i+j}$. Let $d=y^{i j}$. Then

$$
d^{2}=y^{i j} y^{i j}=y^{i} \cdots y^{i} y^{j} \cdots y^{j}=y^{i} \cdots y^{i}=y^{i j}=d
$$

and $d+d=d(d$ is a power of $y)$.

In virtue of the proposition we just proved, immediately after Lemma 2, we could conclude that if $x R=R$ for all elements of a subsemiring $A, R$ has a left unit since $A$ contains at least one multiplicative idempotent. We would therefore show that our assumption was contradictory without resorting to the subsequent lemmas. 
(2) In view of what was proven in this paper, one might raise the following question:

Given a nonoriented graph $G$, under which conditions on $G$ would it be possible to find a semiring $R$ such that $G=G(R)$ ?

And more specifically:

If $D(G) \leqslant 3$, can we find a semiring $R$ such that $G=G(R)$ ?

The answer to the second question is negative.

To prove this, we consider a semiring $R$ and two proper semirings of $R, A$ and $B$, with a nonempty intersection $C$. Then every subsemiring of $R$ which meets $C$ meets $A$ and $B$ as well. Hence, if $G=G(R)$ for some semiring $R, G$ must satisfy the following property:

For every pair of adjacent vertices $a$ and $b$, there is a vertex $c$ (possibly equal to $a$ or to $b$ ) such that for every vertex $d$ which is adjacent to $c$, either $d=a, d=b$, or $d$ is adjacent to both $a$ and $b$.

In the figure below are shown two (of the infinitely many) finite graphs which do not satisfy this property. Hence neither is the graph of any semiring.

$G_{1}$ :

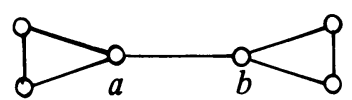

$G_{2}$ :

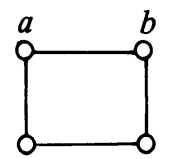

(3) If $G(R)$ is finite, then $R$ is finite. Suppose first that $R$ has no proper subsemiring. Then, if $a \in R,\langle a\rangle=R$. Fix $a$. Since

$$
\langle a\rangle=\left\langle a^{3}\right\rangle=R, \quad a=a^{2} p(a), p(a)
$$

being a sum of powers of $a$.

Let $e=a p(a)$. Then

$$
e^{2}=a p(a) a p(a)=(a a p(a)) p(a)=a p(a)=e .
$$

Since $\langle e\rangle=\langle 3 e\rangle=R$ and $e$ is a multiplicative idempotent, $e=3 k e$ for some positive integer $k$. Let $f=(3 k-1) e$. Then

$$
\begin{aligned}
f+f & =(3 k-1) e+(3 k-1) e=3 k e+(3 k-2) e \\
& =e+(3 k-2) e=(3 k-1) e=f
\end{aligned}
$$

and

$$
\begin{aligned}
f^{2} & =\left(9 k^{2}-6 k+1\right) e=(3 k-2)(3 k e)+e \\
& =(3 k-2) e+e=(3 k-1) e=f .
\end{aligned}
$$

Since $R=\langle f\rangle=\{f\}, R$ is finite.

Suppose that we proved that a semiring is finite if it has less than $k$ proper subsemirings and that $R$ has exactly $k$. Then all proper subsemirings of $R$ will be finite. (Each of them is a semiring having less than $k$ proper subsemirings.) 
Now if $R$ is not cyclic, $R$ is the union of its proper subsemirings. In this case, $R$ is a finite union of finite sets, hence finite.

Suppose then that $R=\langle a\rangle$ for some $a$. We shall prove that $R$ is finite.

Case 1. $a R=R$.

Since $a R=R$ and $R$ is commutative $(R=\langle a\rangle), R$ has a unit $u$. In the sequence $\{n u\}$, at least two terms are equal. Indeed, if this is not the case, $\langle u\rangle$ as well as $\langle 3 u\rangle$ are infinite. Then they cannot be proper subsemirings of $R$ and $\langle u\rangle=\langle 3 u\rangle=R$. Therefore, $u \in\langle 3 u\rangle$ and $u=3 k u$ for some positive integer $k$, a contradiction.

If $k u=(k+m) u$, then for all $x \in R, k x=(k+m) x$. Consider the sequence $\left\{a^{n}\right\}$. If there are repetitions, for instance, $a^{i}=a^{i+j}, R$ is finite. Indeed, all $x \in R$ may, in this case, be written as a sum of powers of $a$ in such a way that:

(a) No exponent exceeds $i+j-1$.

(b) No more than $(k+m-1)$ summands are equal.

Suppose therefore that no two terms are equal in the sequence $\left\{a^{n}\right\}$, and that $c$ is an element of $R$ such that $a c=u(a R=R)$. Then we may not have repetitions in $\left\{c^{n}\right\}$ and $\langle c\rangle$ cannot be a proper subsemiring of $R$ since it is infinite. Therefore, $\langle c\rangle=R$ and $a \in\langle c\rangle$.

Let $a=q(c)$ and suppose that the highest exponent of $c$ in $q(c)$ is $n^{*}$. Then $a^{n^{*}+2}$ is a sum of terms, each of which is a power of $a$ with exponent less than $n^{*}+1$. (Multiply both sides of $a=q(c)$ by $a^{n^{*}+1}$.) Again, all elements of $R$ may be written as sums of powers of $a$ in such a way that:

(a) No exponent exceeds $n^{*}+1$.

(b) No more than $(k+m-1)$ summands are equal.

Case 2. $a R \neq R$.

Then $a R$ is finite.

Consider the sequence $\{n a\}$. If there are repetitions, for instance, $k a$ $=(k+m) a$, put $P=\{n a \mid n=1,2 \cdots, k+m-1\}$. Then $R=\langle a\rangle=P$ $\cup a R \cup(P+a R)$ and $R$ is clearly finite.

Suppose, therefore, that no two terms are equal in $\{n a\}$. Then $\langle n a\rangle$ is infinite for all $n$, and therefore cannot be a proper subsemiring of $R$. So for all $n$, $\langle n a\rangle=R$.

So there is a sequence $\left\{\left(m_{n}, q_{n}(a)\right)\right\}$, such that if $i<j, m_{i}<m_{j}$, and for all $n: a=m_{n} a+a q_{n}(a)$. Indeed, if we know the first $k$ terms of $\left\{\left(m_{n}, q_{n}(a)\right)\right\}$, we put $m=m_{k}+1$. Since $a \in\langle m a\rangle, a=d m a+a q_{k+1}(a)$ for some positive integer $d$, and some $q_{k+1}(a)$. Put $m_{k+1}=d m$. Clearly $m_{k+1}>m_{k}$.

To find $m_{1}$, we consider $\langle 2 a\rangle$. Since $a \in\langle 2 a\rangle$,

$$
a=2 d a+a q_{1}(a) \text { for some } d, q_{1}(a)
$$

We take $m_{1}=2 d$.

The terms $a q_{n}(a)$ belong to $a R$ and $a R$ is finite. Therefore, for some $i$ and $j$, $i<j, a q_{i}(a)=a q_{j}(a)$. Since 


$$
\begin{gathered}
a=m_{i} a+a q_{i}(a) \text { and } a=m_{j} a+a q_{j}(a), \\
\left(\left(m_{j}-m_{i}\right)+1\right) a=\left(m_{j}-m_{i}\right) a+a=\left(m_{j}-m_{i}\right) a+m_{i} a+a q_{i}(a) \\
=m_{j} a+a q_{j}(a)=a .
\end{gathered}
$$

So there are repetitions in $\{n a\}$ and $R$ is finite.

In view of Cases 1 and 2 , it is apparent that by induction on the number of proper subsemirings of $R$, we may prove that if $G(R)$ is finite, $R$ is finite.

(4) Let $G(R)$ be finite. Then $R$ is finite and by Remark 1, all its subsemirings contain idempotents (we use the term "idempotent" for elements which are idempotents for both operations in $R$ ). In this case, if we know $G(R)$, we know the distribution of idempotents in the subsemirings of $R$ as well as the total number of idempotents contained in $R$. Indeed, $A \cap B \neq \varnothing$, if and only if $A$ and $B$ have one or more idempotents in common. (If $A \cap B$ is nonvoid, it is a subsemiring of $R$ and it contains at least one idempotent.) On the other hand, a subsemiring $C$ contains exactly one idempotent, if and only if, for all subsemirings $A$ and $B$ of $R$, the relationships $A \cap C \neq \varnothing$ and $B \cap C \neq \varnothing$ imply $A \cap B \neq \varnothing$.

Therefore, if we know $G(R)$ we may

(a) find the vertices corresponding to proper subsemirings which contain exactly one idempotent,

(b) count the idempotents in $R$,

(c) find the idempotents contained in every subsemiring of $R$,

(d) prove in certain cases that $R$ is not commutative.

For example: If $u, v$ are idempotents of $R$, we may find through $G(R)$ all idempotents contained in $\langle u, v\rangle$. If $\langle u, v\rangle$ has more than six idempotents, $R$ cannot be commutative. For suppose it were. Then since $u^{2}=2 u=u, v^{2}$ $=2 v=v$ and $u v=v u,\langle u, v\rangle$ has at most seven elements: $u, v, u+v, u v$, $u+u v, v+u v, u+v+u v$, all of which, therefore, must be idempotents. On the other hand, $(u+v)^{2}=u+v+u v$ and $(u+v)^{2}=u+v$, hence $\langle u, v\rangle$ has at most six idempotents, a contradiction.

ACKNOWLEDGEMENT. I wish to thank the referee, whose suggestions made this paper intelligible.

\section{REFERENCES}

1. Y.-F. Lin and J. S. Ratti, The graphs of semirings, J. Algebra 14 (1970), 73-82. MR 42 \#3128.

2. The diameters of the graphs of semirings, J. Austral. Math. Soc. 11 (1970), 433-440. MR 44 \#2790.

3. - The graphs of semirings. II, Proc. Amer. Math. Soc. 30 (1971), 473-478. MR 44 \#6772.

4. Anastase Nakassis, Sur la connexité du graphe associé à un demianneau, C.R. Acad. Sci. Paris Sér. A-B 276 (1973), A105-A107. MR 47 \#3466.

4623 Ellicott Street, N.W., Washington D.C. 20016 